\title{
Circular RNA Expression Profiling Identifies Prostate Cancer- Specific circRNAs in Prostate Cancer
}

\author{
Qianlin Xia ${ }^{\mathrm{a}, \mathrm{b}}$ Tao Ding ${ }^{\mathrm{c}}$ Guihong Zhang ${ }^{\mathrm{a}}$ Zehuan Li $^{\mathrm{d}} \quad$ Ling Zeng $^{\mathrm{a}}$ \\ Yanjun Zhue Jianming Guo ${ }^{e}$ Jun Hou ${ }^{f}$ Tongyu Zhu \\ Jianghua Zheng ${ }^{\mathrm{b}}$ Jin Wang ${ }^{\mathrm{a}}$
}

aScientific Research Center, Shanghai Public Health Clinical Center, Fudan University, Jinshan District, Shanghai, bepartment of Laboratory Medicine, Zhoupu Hospital Affiliated to Shanghai University of Medicine \& Health Sciences, Shanghai, 'Department of Urology, the Sixth People's Hospital South Campus, Shanghai Jiao Tong University, Fengxian District, Shanghai, dDepartment of General Surgery, Zhongshan Hospital, Fudan University, Shanghai, eDepartment of Urology, Zhongshan Hospital, Fudan

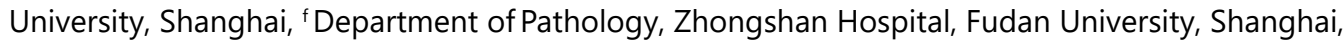
China

\section{Key Words}

Prostate cancer $\bullet \operatorname{circRNAs} \cdot$ Functional network analysis $\bullet$ Biomarkers

\begin{abstract}
Background/Aims: Prostate cancer ( $\mathrm{PCa}$ ) is one of the main cancers that damage males' health severely with high morbidity and mortality, but there is still no ideal molecular marker for the diagnosis and prognosis of prostate cancer. Methods: To determine whether the differentially expressed circRNAs in prostate cancer can serve as novel biomarkers for prostate cancer diagnosis, we screened differentially expressed circRNAs using SBC-ceRNA array in 4 pairs of prostate tumor and paracancerous tissues. A circRNA-miRNA-mRNA regulatory network for the differential circRNAs and their host genes was constructed by Cytoscape3.5.1 software. Quantitative real-time polymerase chain reaction analysis (qRT-PCR) was performed to confirm the microarray data. Results: We found 1021 differentially expressed circRNAs in PCa tumor using SBC-ceRNA array and confirmed the expression of circ_0057558, circ_0062019 and SLC19A1 in PCa cell lines and tumor tissues through qRT-PCR analysis. We demonstrated that combination of PSA level and two differentially expressed circRNAs showed significantly increased AUC, sensitivity and specificity $(0.938,84.5 \%$ and $90.9 \%$, respectively) than PSA alone (AUC of serum PSA was 0.854 ). Moreover, circ_0057558 was correlated positively with total cholesterol. The functional network of circRNA-miRNA-mRNA analysis showed that circ_0057558 and circ_0034467 regulated miR-6884, and circ_0062019 and circ_0060325
\end{abstract}

Q. Xia, T. Ding and G. Zhang contributed equally to this work.

\begin{tabular}{ll}
\hline Jin Wang, Ph.D. & Scientific Research Center, Shanghai Public Health Clinical Center; Fudan Univ., Dept. of Lab. Med., \\
and Jianghua Zheng, Ph.D & Zhoupu Hosp. Aff. to Shanghai Univ. of Med. \& Health Sciences, Shanghai 201508 (China) \\
& Tel. 86-21-57036495, Fax 86-21-57247094, E-Mail wangjin@shaphc.org; zhengjianghua2014@163.com
\end{tabular}


regulated miR-5008. Conclusion: Our results demonstrated that differentially expressed circRNAs (circ_0062019 and circ_0057558) and host gene SLC19A1 of circ_0062019 could be used as potential novel biomarkers for prostate cancer.

\section{Introduction}

Prostate cancer ( $\mathrm{PCa}$ ) is one of the most common cancers in men worldwide, leading high mortality and morbidity, especially for elderly men. It is estimated that about 180, 890 men are diagnosed with prostate cancer in the United States in 2016, with approximately 26, 120 deaths caused by this cancer $[1,2]$. Prostate cancer is a kind of heterogeneous disease which can present as either indolent or aggressive. Although most patients present with localized prostate cancer which is potentially curable by surgery or radiotherapy, up to $30 \%$ men will suffer relapse in 5-10 years, and rapid biochemical recurrence would result in prostate cancer-specific death in $10 \%$ of these patients [3]. The main therapy is prostatectomy for primary prostate cancer and androgen deprivation for metastatic disease, but for castrateresistant prostate cancer, treatment options are limited [4]. Thus, it is important to decide who needs treatments and who does not in order to avoid the unnecessary treatments that may result in severe complications and influence their quality of life without any survival benefits. At present, serum prostate-specific antigen (PSA) remains the standard biomarker for prostate cancer diagnosis and management. But the PSA test often lead to overdiagnosis and overtreatment due to its poor specificity [5], there still is no consensus as to whether this screening test significantly reduces the rate of death from prostate cancer [6]. Moreover, the molecular mechanism of prostate cancer pathogenesis, invasion and metastasis remains largely unknown. It is important to identify the genetic drivers of prostate cancer in order to develop new biomarkers to stratify the risk and aggressiveness of prostate cancer at the time of screening.

Circular RNAs (circRNAs) as non-coding RNAs are endogenous single-stranded circular molecules formed by pre-mRNA back-splicing and characterized by abundant expression, high tissue specificity and stability [7, 8]. Scientists first discovered circRNAs in plantinfected viruses and Sendai virus in the 1970s and subsequently confirmed the extensive expression of circRNAs in fungal yeasts and animal cells [9-11]. Studies investigate that over $10 \%$ of expressed genes can produce circRNAs and the expression level of circRNAs is sometimes 10 times higher than that of their respective host genes [12]. circRNA has a closed ring structure with highly conservative sequence, therefore, it is not easy to be digested by RNase. Moreover, in saliva, blood and other body fluids, circRNAs can also be expressed stably $[13,14]$. circRNAs play roles in the regulation of cell proliferation, apoptosis, angiogenesis and metastasis, suggesting that circular RNAs may involve in tumor formation $[15,16]$ and become novel biomarkers for the diagnosis and treatment of tumor [17]. circ_100290 could play a role of endogenous competitive RNA with the miR-29 family to regulate the expression of CDK6 in the development of oral cancer [18]. circ_0013958 played a role of miR-134 sponge and regulate the expression of cyclin D1 protein in the development of non-small cell lung cancer [19]. circ_100269 is down regulated in gastric cancer and inhibits the growth of gastric cancer cells by targeting miR-630 [20]. Lü investigated that the expression level of circ_103110, circ_104689 and circ_104821 were up-regulated in breast cancer tissues while those of circ_006054, circ_100219 and circ_406697 were down-regulated [21]. With the constant breakthrough and application of new technologies such as chip technology and second-generation sequencing, circular RNA has gradually become the focus of tumor genomics research [22].

However, the differential expression of circRNAs in prostate cancer is rarely reported. Besides, the role of circRNAs in tumorigenesis and progression of prostate cancer remains largely unknown. In order to understand the molecular mechanism of circRNAs in prostate cancer, herein we investigated the differentially expressed profiles of circRNAs in prostate cancer using SBC Human competing endogenous RNAs (ceRNA) microarray and verified the 
differential circRNAs in prostate cells and tissues. We also analyzed the correlation between differential circRNAs and clinical parameters in order to find new targets for clinical diagnosis and treatment of prostate cancer. Our exploration for circRNAs will help to add a new dimension to the study of molecular mechanisms of prostate cancer and will also provide new directions for cancer diagnosis and treatment.

\section{Materials and Methods}

\section{Cell culture and Prostate Cell lines}

The prostate cancer cell lines 22RV1 (ATCC Number: CRL-2505), DU145 (ATCC Number: HTB-81), Lncap (ATCC Number: CRL-1740) and PC3 (ATCC Number: CRL-1435) were purchased from the Culture Collection of the Chinese Academy of Sciences, Shanghai, China (http://www.cellbank.org.cn/). DU145 and PC3 were cultured in MEM (GIBCO, 41500034, Life Technologies) and F-12 (GIBCO, 21700075, Life Technologies), respectively; LNCAP and 22RV1 were maintained in RPMI-1640 (GIBCO, 31800022, Life Technologies); supplemented with $10 \%$ fetal bovine serum (FBS) (Invitrogen, GIBCO) at $37^{\circ} \mathrm{C}$ in $5 \%$ C02. The human prostatic epithelial cell lines (HPEpic) were purchased from Shanghai Xinyu Biological Technology Co., Ltd. All cells were cultured according to ATCC standard procedure.

\section{Prostate tumor and benign prostatic hyperplasia (BPH) tissue samples}

Four pairs of prostate cancer and paracancerous tissues undergoing radical prostatectomy, and 173 cases of prostate puncture tissues including 95 cases of prostate cancer tissues and 78 cases of BPH tissues were acquired from Zhongshan Hospital Affiliated to Fudan University. This research was approved by The Ethics Committee of Zhongshan Hospital Affiliated to Fudan University and Shanghai Public Health Clinical Center. Study populations provided written informed consent. Each tissue was confirmed by a pathologist specializing in prostate cancer, and a Gleason score was provided to measure risk stratification. All samples were stored at $-80 \mathrm{C}^{\circ}$ after surgical resection.

\section{RNA purification, SBC ceRNA Microarray, and Data analysis}

Total RNA was extracted and purified using Trizol reagent (Invitrogen) and RNeasy Mini Kit (Qiagen, USA) following the manufacturer's instructions. Total RNA was quantified by NanoDrop-2000 spectrophotometer (NanoDrop, USA), and assessed by Agilent Bioanalyzer 2100 (CA, US) to inspect RNA integration. SBC ceRNA microarray covers 88,371 circRNAs, 68,423 IncRNAs and 18, 853 mRNAs. Four pairs of prostate cancer and paracancerous tissues were used for microarray assay to investigate the differentially expressed circRNAs between cancer tissues and paracancerous tissues. Total RNA was amplified and labeled by Low Input Quick Amp WT Labeling Kit (Santa Clara, CA, US), Labeled cRNAs were purified by RNeasy mini kit. The microarray hybridization was performed following the manufacturer's standard protocols using Gene Expression Hybridization Kit (Santa Clara, CA, US) in Hybridization Oven (Santa Clara, CA, US). The hybridized slides were washed, fixed and finally scanned to images by Agilent Microarray Scanner (Santa Clara, CA, US). Data were extracted with Feature Extraction software 10.7 (CA, US), raw data were normalized by Quantile algorithm, limma packages in R. Significant differentially expressed circRNA between prostate cancer and paracancerous tissues were identified and retained by screening fold change $>2.0, p<0.05$.

\section{Functional network analysis for the differential circRNAs and their host genes}

To understand the role of differentially expressed circRNAs, Gene Ontology (GO) analysis and KEGG (Kyoto Encyclopedia of Genes and Genomes) were performed to construct meaningful annotation of genes as a whole network [23]. At the same time, we also constructed a circRNA-miRNA-mRNA network in transcripts. By analyzing the possible combination of circRNAs and miRNA, we predicted the target miRNAs of 100 circRNAs with the largest differences in ceRNA chip and draw a circRNA-miRNA regulatory network map. Then, we selected circRNAs that were verified to be differentially expressed in prostate cancer to further predict their target miRNAs and draw a circRNA-miRNA-mRNA regulatory network map by Cytoscape3.5.1 software. The miRNA binding sites of circRNAs were predicted by miRcode (http:// www.mircode.org/), while the potential miRNA response elements of mRNA were predicted by Targetscan (http://www.targetscan.org/). 


\section{Cellular Physiology Cell Physiol Biochem 2018;50:1903-1915

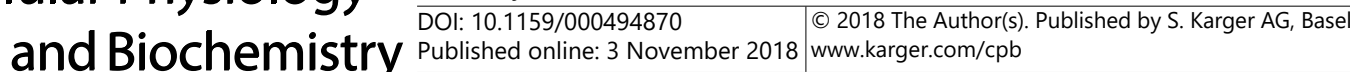 \\ Xia et al.: circRNAs Profiling in Prostate Cancer}

Quantitative real-time polymerase chain reaction analysis ( $q R T-P C R$ )

173 cases of prostate puncture tissues as well as four prostate cancer cell lines (22RV1, DU145, Lncap and PC3) and a human prostatic epithelial cell lines (HPEpic) were used for qRT-PCR validation. After RNA isolation, PrimeScript $^{\mathrm{TM}}$ RT reagent Kit with gDNA Eraser (TaKaRa) was used to synthesize cDNA according to the manufacturer's instructions. The kit SYBR Premix Ex Taq ${ }^{\mathrm{TM}}$ II (TaKaRa) was used to detect the expression level of circRNA. We performed qRT-PCR in a total reaction volume of $20 \mu \mathrm{l}$, including $10 \mu \mathrm{l}$ of $2 \times$ SYBR Green PCR buffer, $0.8 \mu \mathrm{l}$ of Forward primer (10 $\mu \mathrm{M}), 0.8 \mu \mathrm{l}$ Reverse primer $(10 \mu \mathrm{M}), 0.4 \mu \mathrm{l}$ of ROX reference Dye, $7 \mu \mathrm{l}$ of $\mathrm{ddH}_{2} \mathrm{O}$ and 30 ng cDNA. The reaction was initiated at $95^{\circ} \mathrm{C}$ for $10 \mathrm{~min}$, then at $95^{\circ} \mathrm{C}(15 \mathrm{~s}), 60^{\circ} \mathrm{C}(60 \mathrm{~s})$ for a total 40 cycles. $18 \mathrm{~S}$ was used as an internal reference. Results were acquired from three independent wells. The relative expression level was calculated using $2^{-\Delta \Delta \mathrm{ct}}$ method. Student's t-tests were performed, and $\mathrm{p}<0.05$ was considered to be significant. The values were described as means \pm SD.

\section{Statistical analyses}

We collected clinical data of the 173 prostate tissues and student's t-test was used to analyze the difference of circRNAs expression between prostate cancer group and $\mathrm{BPH}$ group. Pearson correlation analysis was used to investigate the relationship between differential circRNAs and clinical parameters. The results were regarded as statistically significant at $\mathrm{p}<0.05$. All graphs were built using GraphPad Prism 5.0 software (GraphPad Software Inc., La Jolla, CA, USA). Statistical analysis was performed using the SPSS 20.0 (IBM-SPSS Inc., Chicago, IL, USA). Receiver operating characteristic (ROC) curves were applied to evaluate the clinical diagnostic value of differential circRNAs and the combination of PSA and circRNAs.
Table 1. Partially differentially expressed circRNAs and their corresponding host genes (cancer/paracancerous tissue)

\begin{tabular}{|c|c|c|c|c|}
\hline Probe Name & Best_transcript & Fold chang & $\mathrm{p}$ values & Host gene \\
\hline circ_0057553 & NM_001127257 & 5.013 & 0.044 & SLC39A10 \\
\hline circ_0057555 & NM_001127257 & 4.282 & 0.038 & SLC39A10 \\
\hline circ_0074032 & NM_145282 & 4.243 & 0.004 & SLC25A48 \\
\hline circ_0057554 & NM_001127257 & 4.139 & 0.041 & SLC39A10 \\
\hline circ_0057558 & NM_001127257 & 4.103 & 0.048 & SLC39A10 \\
\hline circ_0057559 & NM_001127257 & 4.059 & 0.037 & SLC39A10 \\
\hline circ_0017076 & NM_001098722 & 3.837 & 0.024 & GNG4 \\
\hline circ_0006813 TC & CONS_12_00022658 & 3.311 & 0.020 & TCONS_12_00022658 \\
\hline circ_0056881 & NM_001935 & 3.198 & 0.005 & DPP4 \\
\hline circ_0062019 & NM_194255 & 3.140 & 0.014 & SLC19A1 \\
\hline circ_0046306 & NM_016286 & 3.076 & 0.024 & DCXR \\
\hline circ_0062018 & NM_194255 & 2.983 & 0.012 & SLC19A1 \\
\hline circ_0060919 & NM_003657 & 2.933 & 0.035 & BCAS1 \\
\hline circ_0062020 & NM_194255 & 2.932 & 0.017 & SLC19A1 \\
\hline circ_0036682 & NM_007011 & 2.912 & 0.046 & ABHD2 \\
\hline circ_0046302 & NM_016286 & 2.902 & 0.026 & DCXR \\
\hline circ_0029885 & NM_003045 & 2.805 & 0.040 & SLC7A1 \\
\hline circ_0035036 & NM_025165 & 2.780 & 0.031 & ELL3 \\
\hline circ_0046304 & NM_016286 & 2.732 & 0.036 & DCXR \\
\hline circ_0092226 & NM_033284 & 2.674 & 0.021 & TBL1Y \\
\hline circ_0004529 & NM_020066 & -3.711 & 0.006 & FMN2 \\
\hline circ_0034539 & NM_003246 & -3.720 & 0.010 & THBS1 \\
\hline circ_0034512 & NM_003246 & -3.722 & 0.012 & THBS1 \\
\hline circ_0034531 & NM_003246 & -3.725 & 0.011 & THBS1 \\
\hline circ_0034506 & NM_003246 & -3.739 & 0.009 & THBS1 \\
\hline circ_0091784 & NM_000425 & -3.809 & 0.004 & L1CAM \\
\hline circ_0066445 & NM_182920 & -3.877 & 0.011 & ADAMTS9 \\
\hline circ_0034480 & NM_003246 & -3.889 & 0.004 & THBS1 \\
\hline circ_0034464 & NM_003246 & -3.906 & 0.006 & THBS1 \\
\hline circ_0030845 & NM_001845 & -4.046 & 0.013 & COL4A1 \\
\hline circ_0034465 & NM_003246 & -4.062 & 0.005 & THBS1 \\
\hline circ_0078704 & NM_003247 & -4.097 & 0.018 & THBS2 \\
\hline circ_0060328 & NM_004613 & -4.200 & 0.035 & TGM2 \\
\hline circ_0003924 & NM_006902 & -4.517 & 0.013 & PRRX1 \\
\hline circ_0034470 & NM_003246 & -4.521 & 0.020 & THBS1 \\
\hline circ_0075538 & NM_000129 & -5.008 & 0.028 & F13A1 \\
\hline circ_0075540 & NM_000129 & -5.427 & 0.014 & F13A1 \\
\hline circ_0075539 & NM_000129 & -5.652 & 0.017 & F13A1 \\
\hline circ_0075542 & NM_000129 & -6.379 & 0.028 & F13A1 \\
\hline circ_0075541 & NM_000129 & -7.066 & 0.005 & F13A1 \\
\hline
\end{tabular}

\section{Results}

Differential profiling of circRNAs and genes in prostate tumor

The probes of ceRNA microarray have been firstly used to detect a large number of transcripts in prostate cancer and corresponding paracancerous tissues. Scatter plots (Fig. 1A, B) and heatmap (Fig. 1C, D) of differential circRNAs and mRNAs in prostate tumor tissues and normal tissues were shown in Fig. 1, The heatmap indicated that a total of 1021 circRNAs (Fig. 1C) and 250 mRNAs (Fig. 1D) were differentially expressed with fold change $>2.0$, p < 


\section{Cellular Physiology Cell Physiol Biochem 2018;50:1903-1915

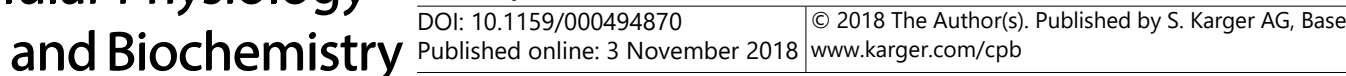

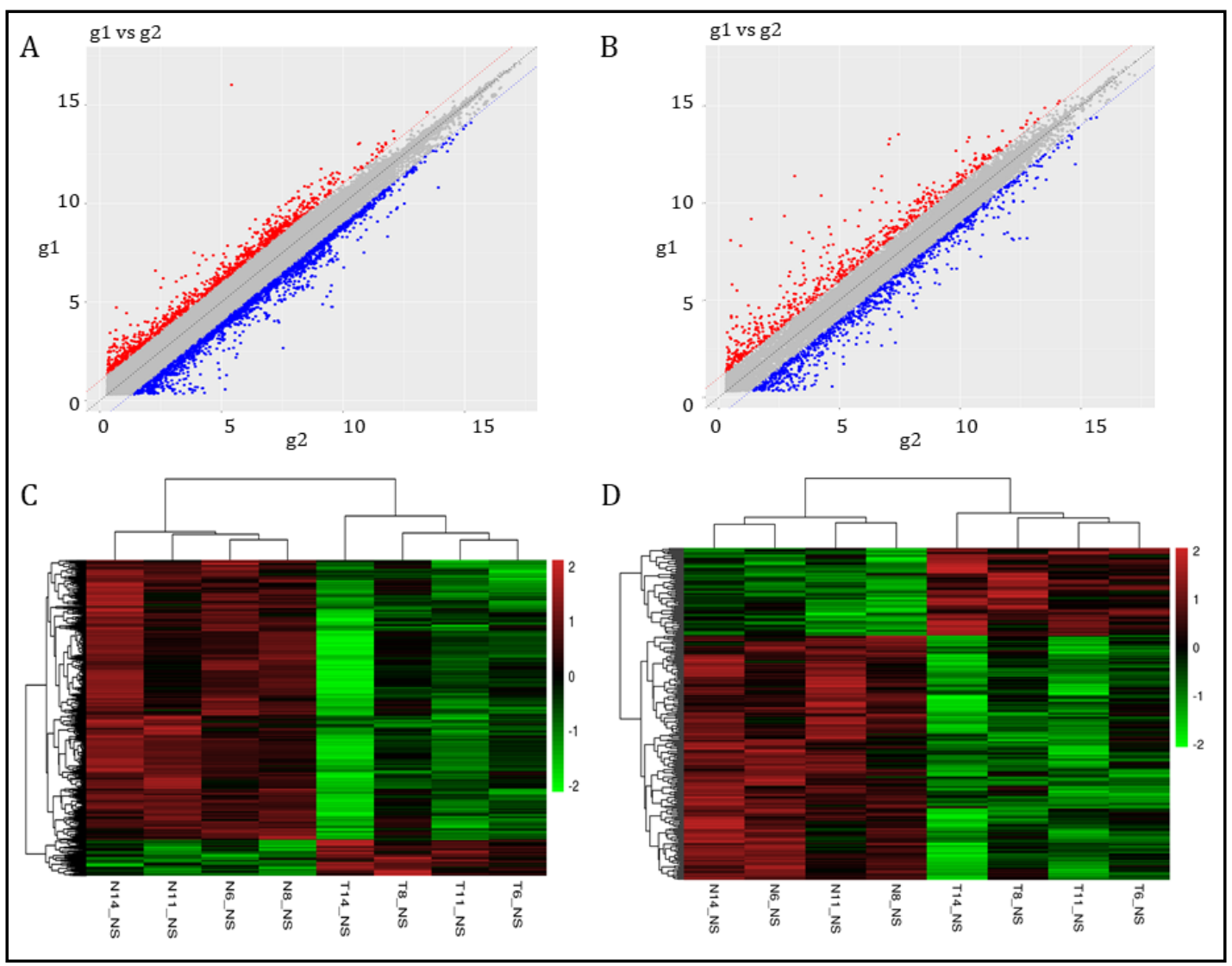

Fig. 1. Scatter plots and heatmap of differential circRNAs and mRNA in prostate tumor tissues and normal tissues. A, B) the scatter plots of differential circRNAs (A) and mRNA (B) in ceRNA microarray; C, D) the heatmap of differential circRNAs (C) and mRNA (D).

0.05. Among them, 117 circRNAs and 66 mRNAs were upregulated, and 904 circRNAs and 184 mRNAs were dowregulated in four pairs of prostate cancer /adjacent tissues (Table 1). Among the differential profiling of circRNAs, the most upregulated circRNA is circ_0057553 and the most downregulated circRNA is circ_0075541 which were produced by their host coding genes SLC39A10 and F13A1, respectively.

Functional pathway analysis of differential circRNAs, their host genes and regulatory networks

We predicted the functions of the differentially expressed circRNAs in prostate tumour through GO pathway enrichment analysis of their host genes and demonstrated that the host genes of these circRNAs are mainly involved in biological processes such as response to gonadotropin, trabecula morphogenesis, peptide cross-linking, regulation of blood coagulation and leukocyte cell-cell adhesion; the molecular functions of these host genes are mainly enriched in extracellular matrix (ECM) binding, ECM structural constituent, protease binding, growth factor binding and integrin binding; the cellular components of these host genes were involved in platelet alpha granule, ECM, external side of plasma membrane and adheren junction. The top 30 of GO analysis of these host genes of the 1021 differentially expressed circRNAs are shown in Fig. 2. 


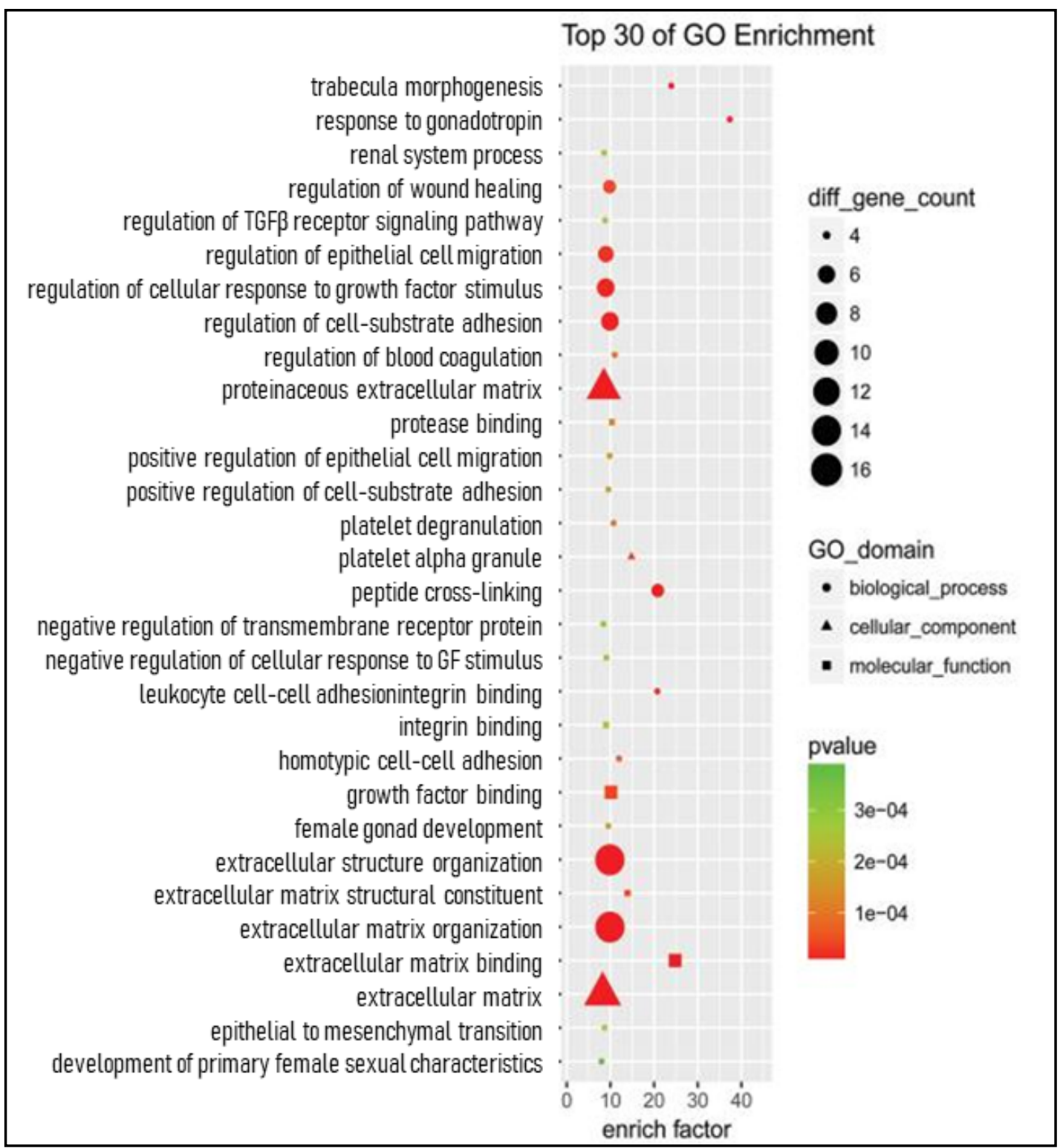

Fig. 2. GO analysis of host genes of differential circRNAs in prostate cancer.

Table 2. The prediction results for target miRNAs of 4 different circRNAs

\begin{tabular}{llllll}
\hline circRNAs & circRNAs_MRE1 & circRNAs_MRE2 & circRNAs_MRE3 & circRNAs_MRE4 & circRNAs_MRE5 \\
\hline circ_0062019 & miR-6838-5p & miR-424-5p & miR-15b-5p & miR-16-5p & miR-5008-5p \\
circ_0057558 & miR-6894-3p & miR-6499-3p & miR-6884-3p & miR-7843-3p & miR-4763-5p \\
circ_0034467 & miR-486-3p & miR-6721-5p & miR-3678-3p & miR-6884-3p & miR-1273h-3p \\
circ_0060325 & miR-7155-5p & miR-5008-5p & miR-6722-3p & miR-5787 & miR-1909-3p \\
\hline
\end{tabular}

Further, the target miRNAs of 100 circRNAs with the largest difference in ceRNA chip were predicted and five miRNAs with the most binding sites for each circRNAs were selected for subsequent analysis. The results showed that a total of 317 miRNAs were predicted from 100 differential circRNAs. On the other hand, circRNAs contain microRNA binding sites and act as miRNA sponges, thereby eliminating miRNAs' inhibitory effects on their target genes and increasing the target mRNA expression levels. Based on this endogenous competition mechanism, we first predicted the potential target miRNAs of circ_0057558, circ_0034467 


\section{Cellular Physiology

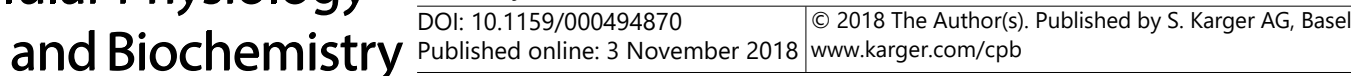

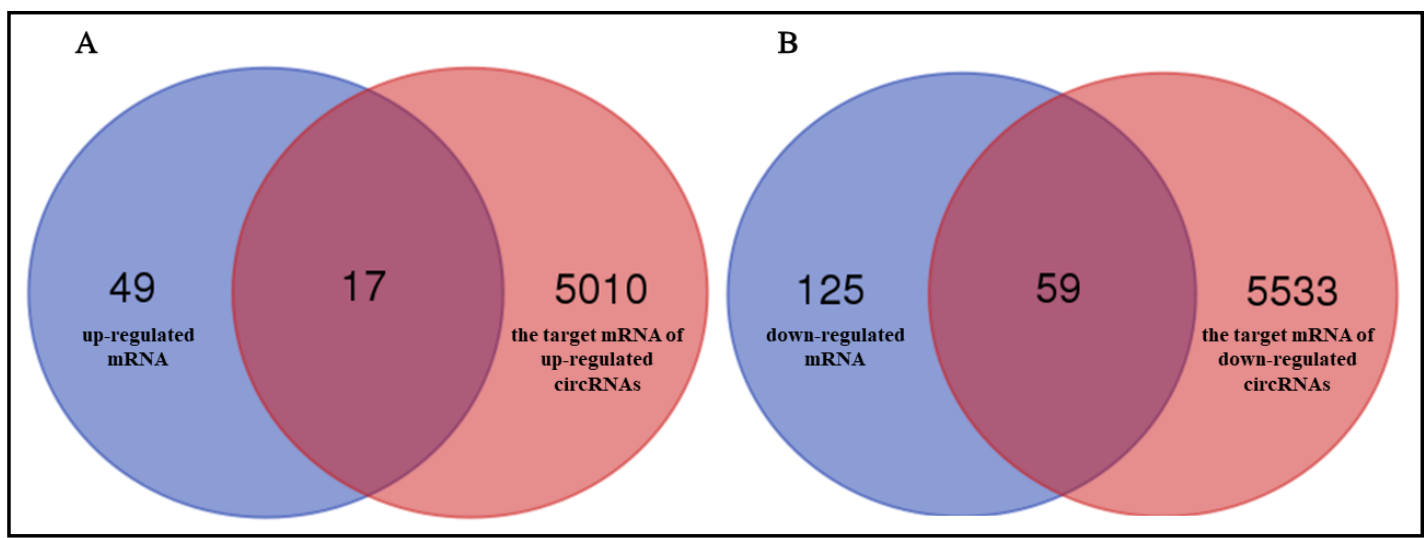

Fig. 3. Comparative analysis of mRNA expression profiles and target genes of differential circRNAs associated miRNAs.

circ_0062019, and circ_0060325 (Table 2). Further, the target genes of these miRNAs were also predicted by IPA. Combining with the mRNA data of our prostate cancer profiling, we identified that 76 mRNAs including 17 upregulated and 59 down-regulated genes as targets of these miRNAs were differentially expressed in prostate tumor (Fig. 3). To obtain insights into reciprocal circRNAs and mRNA regulation, a regulatory network of circRNAs-miRNAmRNA is constructed using the cytoscape software (Fig. S1A - for all supplemental material see www.karger.com/10.1159/000494870/), which includes 4 circRNAs (circ_0057558, circ_0034467 circ_0062019, and circ_0060325), 12 related miRNAs (miR-15b-5p, miR-4245p, miR-486-3p, miR-1273h-3p, miR-3678-3p, miR-4763-5p, miR-5008-5p, miR-6499-3p, miR-6721-5p, miR-6838-5p, miR-6884-3p, and miR-7843-5p,) and 76 target genes. We found that circ_0057558 and circ_0034467 can act together on miR-6884-3p to regulate 8 target genes; circ_0062019 and circ_0060325 work together on miR-5008-5p to regulate 21 target genes. It is noteworthy that the host gene SLC19A1 of circ_0062019 is also involved in this regulatory network. Thus, the specific regulatory patterns of circ_0062019-miR-50085p - SLC19A1, circ_0060325 - miR-5008-5p - SLC19A1 and circ_0034467 - miR-6721-5p - SLC19A1 may be key regulators in prostate cancer (Fig. S1B).

Validation of key differentially expressed circRNAs (circ_0075538, circ_0057558, and circ_0062019) and the host gene SLC19A1

We further verified the related differential circRNAs such as circ_0057558, circ_0062019, circ_0075538, and the host gene SLC19A1 in prostate cancer cells (PCa 22RV1, DU145, Lncap and PC3 cells), normal prostate cell HPEpiC and 173 prostate tissues ( 95 prostate cancer tissues and 78 BPH tissues) by qRT-PCR. The results revealed that circ_0057558 (Fig. 4A) and circ_0062019 (Fig. 4B) were up-regulated in four prostate cancer cells, which was consistent with the results of microarray. In addition, circ_0075538 is down-regulated in PC3 and 22RV1 cells (Fig. 4C). At the same time, we found that SLC19A1 which is the host gene of circ_0062019 was also up-regulated in prostate cancer cells (Fig. 4D). We further validated these circRNAs and SLC19A1 in 95 prostate cancer tissues and $78 \mathrm{BPH}$ tissues. The results showed that circ_0057558 (Fig. 4E) and circ_0062019 (Fig. 4F) were up-regulated in prostate cancer tissues $(\mathrm{p}<0.01)$. We also found that the host gene SLC19A1 was also up-regulated in PCa tissues ( $\mathrm{p}<0.01$ ) (Fig. 4H), which further confirmed the results of our ceRNA microarray although circ_0075538 was down-regulated in prostate cancer $(\mathrm{p}=$ 0.296) (Fig. 4G). 


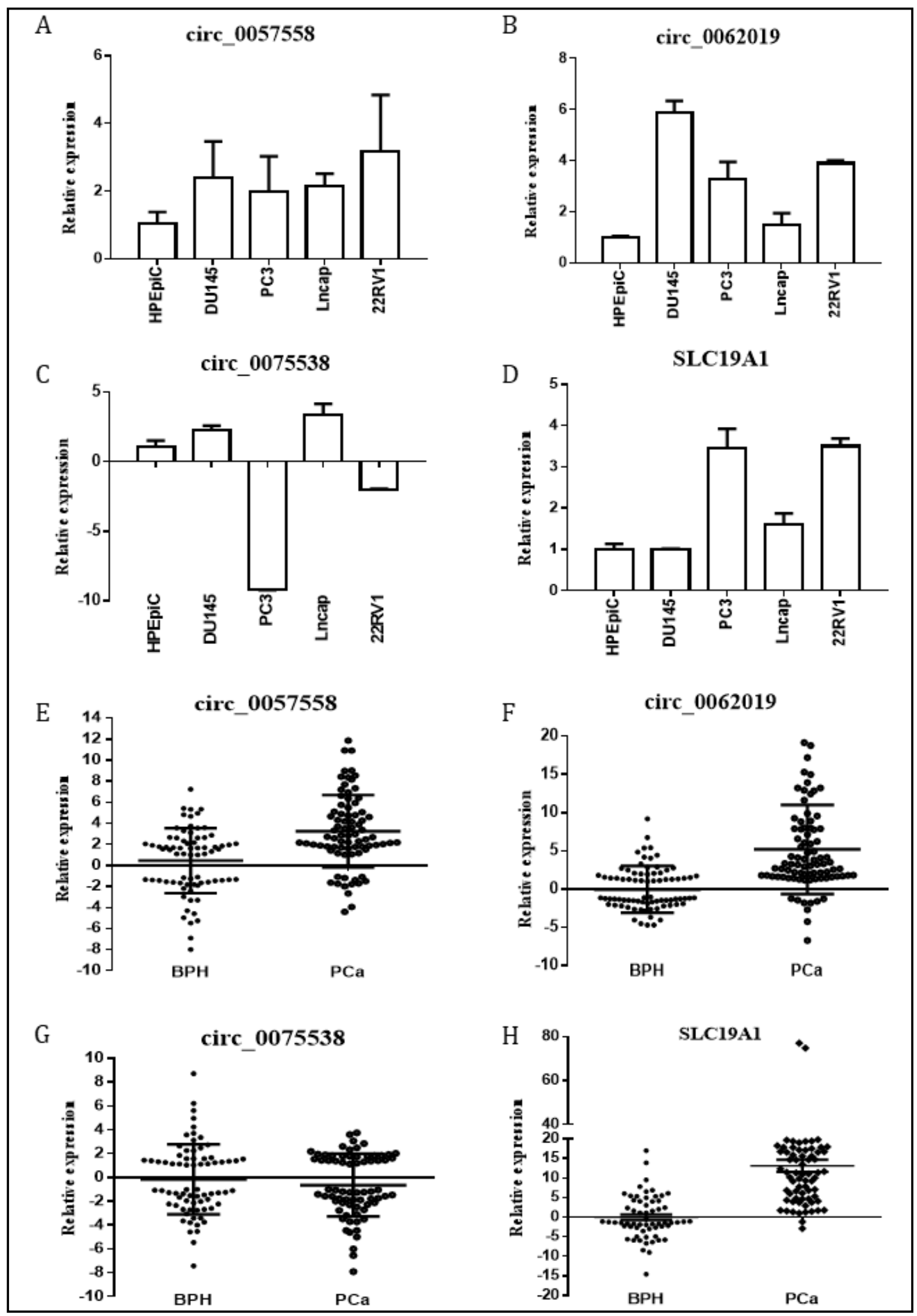

Fig. 4. qRT-PCR analysis of the gene expression levels of circRNAs. circ_0057558 (A, E), circ_0062019 (B, F), circ_0075538 (C, G) and SLC19A1 (D, H) in prostate cells (A-D), and tumor tissue samples compared to BPH tissue samples (E-H). 


\section{Cellular Physiology Cell Physiol Biochem 2018;50:1903-1915 \begin{tabular}{ll|l} 
and Biochemistry Published online: 3 November 2018 & $\begin{array}{l}\text { (c) } 2018 \text { The Author(s). Published by S. Karger AG, Base } \\
\text { www.karger.com/cpb }\end{array}$ \\
\hline
\end{tabular}
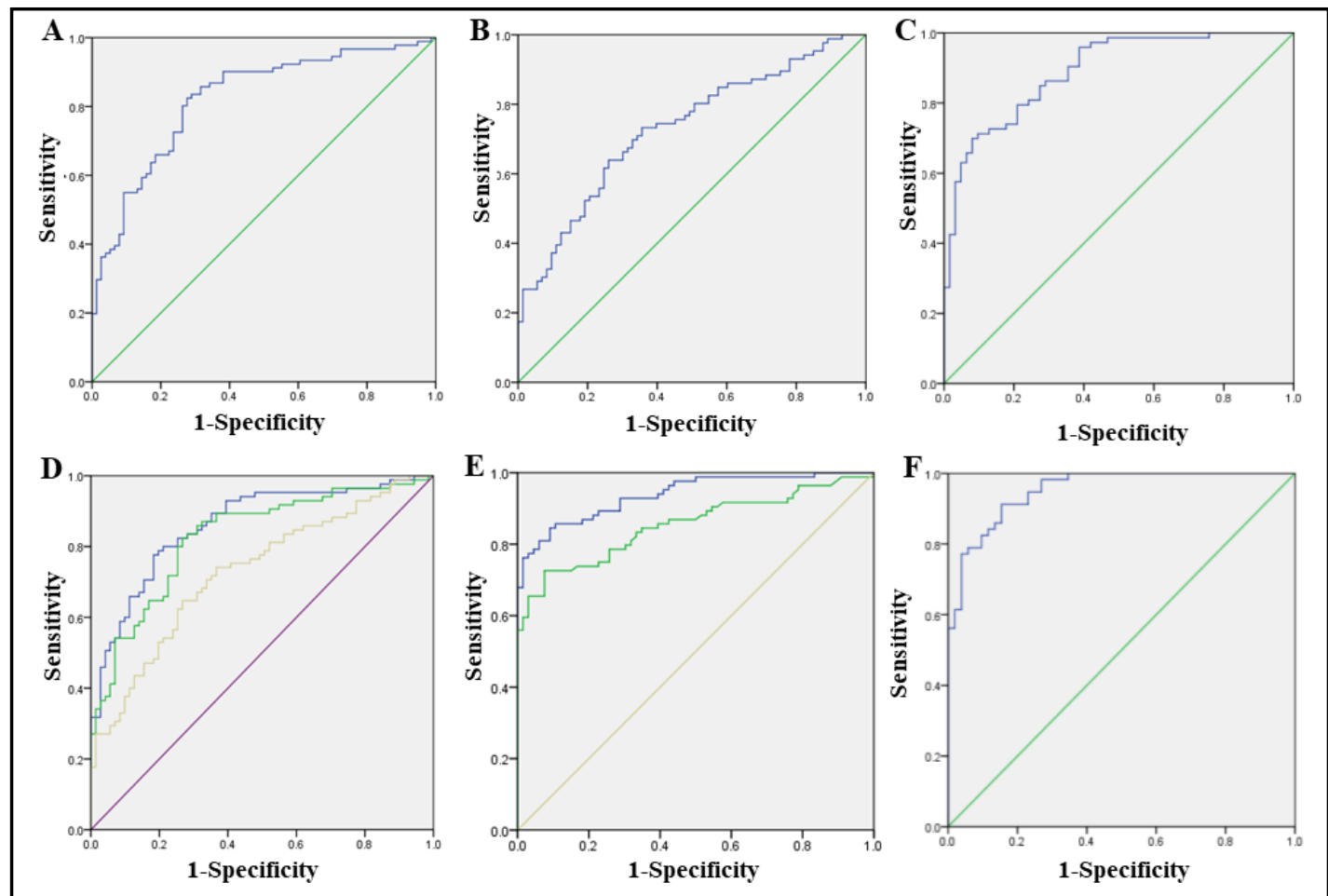

Fig. 5. ROC curve showing expression levels of differential circRNAs. circ_0062019 and circ_0057558: (A, B) and the host gene SLC19A1 (C) in PCa patients and BPH controls; the 2 circRNAs combination (D), the 2 circRNAs and PSA combination (E), the 2 circRNAs, PSA and SLC19A1 combination (F). The R0C curves were analyzed using univariate (log-rank) analysis.

Differentially expressed circRNAs as novel biomarkers of prostate cancer associated with lipid metabolism, serum PSA levels and the progression of PCa

We assessed the diagnostic effectiveness of differential circRNAs to differentiate between prostate cancer and BPH tissues by ROC curve (Fig. 5). The area under the curve (AUC) of circ_0062019, circ_0057558 and SLC19A1 were 0.828, 0.729 and 0.891, respectively (Fig. $5 A-C$, and Table 3). When two differential circRNAs were combined, the AUC is much better (0.861, Fig. 5D) than PSA alone (which AUC of serum PSA was 0.854) although PSA is the main clinical indicator for early screening and diagnosis of prostate cancer, which demonstrated that different levels of these two circRNAs could help distinguish PCa patients from non-PCa patients and combing them could improve the diagnostic efficiency. We further analyzed the PSA level with the results of the 2 differential circRNAs. The results showed that the area of AUC was 0.938 , the sensitivity was $84.5 \%$ and the specificity was $90.9 \%$ (Fig. 5E). When we combined the circRNAs, PSA and SLC19A1, the AUC was up to 0.952 (Fig. 5F). To clarify the characteristics these two differential circRNAs in prostate cancer, a Pearson correlation analysis was applied to analyze the correlation between these circRNAs and the corresponding clinical parameters. As shown in Table 4, circ_0062019 is positively correlated with the age of the patients. circ_0062019 was also positively correlated with the patient's PSA level and Gleason score $(p<0.05)$. A combined Gleason score of 6 or 7 indicates PCa is likely to grow but may not spread quickly. A score of 8-10 means aggressive cancer which is the potentially lethal form of prostate cancer [24]. In this study, we investigated that the associate between the expression of circ_0062019, circ_0075538 and aggressive cancer (Gleason score 8-10, p < 0.05) (Table 5), which indicates that the two circRNAs may be related to the progression of prostate cancer. We also found that circ_0057558 was positively correlated with total cholesterol $(\mathrm{p}=0.04)$ (Table 4), suggesting that circ_0057558 may be associated with lipid metabolism in prostate cancer patients. 


\section{Discussion}

Prostate cancer is one of the most common cancers in developed countries such as Europe and the United States, and its mortality rate ranks the second in male malignancy [25]. The biological characteristics of PCa are complex and its early clinical symptoms are not severe enough to be noticed. At present, clinicians have limited indicators for the screening of prostate cancer among which the most commonly used one is PSA. However, the specificity of PSA is relatively low since it is also elevated in many benign prostate diseases. In addition, PSA is susceptible to many factors and lacks stability $[13,26]$. Therefore, there is an urgent need to find new prostate cancer diagnostic markers for early screening of prostate cancer. In this study, four pairs of PCa and their corresponding paracancerous tissues were analyzed using ceRNA microarrays. A total of 1021 differential circRNAs including 117 up-regulated circRNAs and 904 down-regulated circRNAs were found in PCa tumor tissue. Next, we predicted the function of circRNAs by GO analysis for the host genes of the differential circRNAs in the chip. We found that the enrichment factor of gonadotropic response is the highest, involving genes such as NOTCH1, ICAM1, ITGA3 and TOX2 and related circRNAs such as circ_0089549, circ_0049237, circ_0044457, and circ_0008117. To further understand the role of differentially expressed circRNAs in PCa, we used circRNAs-miRNAmRNA network maps to predict the regulatory networks of circRNAs. We demonstrated that circ_0057558 and circ_0034467 work together on miR-6884-3p to regulate target genes such as AQP1, TUBA1A, LPAR1, EMP1, DHTK1, GINS2, DI03 and ITK; circ_0062019 and circ_0060325 act together on miR-5008-5p to regulate target genes such as CSF1, RAB3IL1, TGM2, GPR68, BIBD11, SIPR4, TGFB1I1, NUDT8, GPT2, while NPTX2. AQP1, LPAR1, TGFB1I1, CSF1 and NPTX2 have been reported in prostate cancer $[7,27]$. It is of note that the target miRNA of circ_0057558 is miR-6884-3p. Although miR-6884-3p has not yet been reported, we analyzed the signal pathway database Reactome (http://www.reactome.org) and found that miR-6884-3p is involved in the metabolism of fatty acids, glycerol and ketone bodies and in triacylglycerols synthesis. We also found that circ_0057558 was significantly positively correlated with triglyceride levels, which suggested that circ_0057558 may regulate lipid metabolism in PCa by regulating miR-6884-3p.

qRT-PCR showed that circ_0062019 and circ_0057558 were up-regulated in four PCa cells, which were in full agreement with the results of the chip, suggesting that these circRNAs are highly expressed in the PCa. circ_0075538 was down-regulated only in PC3 and 22RV1 cells, which did not fully represent their expression level in prostate cells in vitro and needs further verification. In addition, we found that the host gene SLC19A1 of circ_0062019 was up-regulated in our ceRNA chip and also verified the gene in all the four prostate cells. The membrane protein encoded by SLC19A1 is a folate transporter which regulates the 


\section{Cellular Physiology Cell Physiol Biochem 2018;50:1903-1915 \begin{tabular}{ll|l} 
and Biochemistry & $\begin{array}{l}\text { DOl: 10.1159/000494870 } \\
\text { Published online: } 3 \text { November } 2018\end{array}$ & $\begin{array}{l}\text { 2018 The Author(s). Published by S. Karger AG, Basel } \\
\text { www.karger.com/cpb }\end{array}$ \\
\cline { 2 - 3 }
\end{tabular} \\ Xia et al.: circRNAs Profiling in Prostate Cancer}

concentration of folate in cells. A few studies have verified that high levels of folic acid can promote the occurrence and development of PCa [28-30]. Tomaszewski collected serum folic acid levels in patients with PCa and analyzed the relationship between Ki67 and the level of serum folate and found that the proportion of Ki67 positive cells increased significantly in patients with high folate level, indicating that the level of folate in patients was related to the proliferation of PCa cells [31]. Therefore, SLC19A1 and its related circ_0062019 probably affect the progression of PCa by regulating the level of folate in vivo. We also validated that the expression of circ_0057558 and circ_0062019 and its host gene SLC19A1 were significantly up-regulated in PCa tissues $(\mathrm{p}<0.01)$, which was in agreement with the cell verification results, indicating that the two circRNAs are likely to play an important role in the pathogenesis of PCa.

Changes in lipid composition can influence membrane fluidity, cell growth and resistance to chemotherapeutic agents [32,33]. PCa is a hormone-dependent cancer and the growth of cancer cells rely mainly on lipid oxidation [34]. It has been reported that there are lipid metabolism abnormalities in PCa patients and the lipid metabolism process is one of the major targets of PCa cell androgens since it is closely related to the growth, survival and drug resistance of PCa cells [35, 36]. Circ_0057558 was verified to be up-regulated in PCa and positively correlated with total cholesterol, which suggesting that circ_0057558 may promote the growth of PCa through the regulation of lipid metabolism in PCa patients. ROC curves were used to further evaluate the clinical diagnostic value of the two differential circRNAs. The results showed that the AUC of circ_0057558 and circ_0062019 was 0.729 and 0.828 , respectively, Combined detection of circ_0057558 and circ_0062019 significantly improved the diagnostic specificity, sensitivity and AUC $(0.824,0.746$ and 0.861 , respectively) which were superior to those of PSA, through which we can better discriminate PCa from BPH.

\section{Conclusion}

In this study, we have screened two PCa related circRNAs- circ_0057558 and circ_0062019 in PCa. We found that when combining these two circRNAs with PSA, we can effectively distinguish between PCa and BPH patients. Therefore, these two differential circRNAs are expected to become potential biomarkers for PCa screening and diagnosis. Of note, the relationship between the differences in circRNAs and lipid metabolism need further investigation and verification with larger sample size to decide whether they can be applied in clinical practice for PCa.

\section{Acknowledgements}

This research was supported by grants from the National Natural Science Foundation of China (81672383, 81372318), a grant (16PJ1408800) from the Shanghai Pujiang Program, Shanghai, and a grant (PWRL2017-07) supported by Pudong New District Commission of Health and Family Planning leading talent Program (Jianghua Zheng), Shanghai, China.

\section{Disclosure Statement}

The authors have declared that no competing interest exists. 


\section{Cellular Physiology Cell Physiol Biochem 2018;50:1903-1915 and Biochemistry Published online:3November $2018 \begin{aligned} & \text { (c) } 2018 \text { The Author(s). Published by S. Karger AG, Basel } \\ & \text { www.karger.com/cpb }\end{aligned}$}

Xia et al.: circRNAs Profiling in Prostate Cancer

\section{References}

1 Siegel RL, Miller KD, Jemal A: Cancer statistics, 2016. CA Cancer J Clin 2016;66:7-30.

2 Shan M, Xia Q, Yan D, Zhu Y, Zhang X, Zhang G, Guo J, Hou J, Chen W, Zhu T, Zhang X, Xu J, Wang J, Ding T, Zheng J: Molecular analyses of prostate tumors for diagnosis of malignancy on fine-needle aspiration biopsies. Oncotarget 2017;8:104761-104771.

-3 Buyyounouski MK, Pickles T, Kestin LL, Allison R, Williams SG: Validating the interval to biochemical failure for the identification of potentially lethal prostate cancer. J Clin Oncol 2012;30:1857-1863.

4 Masson S, Bahl A: Metastatic castrate-resistant prostate cancer: dawn of a new age of management. BJU Int 2012;110:1110-1114.

-5 Draisma G, Etzioni R, Tsodikov A, Mariotto A, Wever E, Gulati R, Feuer E, de Koning H: Lead time and overdiagnosis in prostate-specific antigen screening: importance of methods and context. J Natl Cancer Inst 2009;101:374-383.

6 Schroder FH, Hugosson J, Roobol MJ, Tammela TL, Zappa M, Nelen V, Kwiatkowski M, Lujan M, Maattanen L, Lilja H, Denis LJ, Recker F, Paez A, Bangma CH, Carlsson S, Puliti D, Villers A, Rebillard X, Hakama M, Stenman UH, Kujala P, Taari K, Aus G, Huber A, van der Kwast TH, van Schaik RH, de Koning HJ, Moss SM, Auvinen A: Screening and prostate cancer mortality: results of the European Randomised Study of Screening for Prostate Cancer (ERSPC) at 13 years of follow-up. Lancet 2014;384:2027-2035.

7 Leach DA, Need EF, Trotta AP, Grubisha MJ, DeFranco DB, Buchanan G: Hic-5 influences genomic and nongenomic actions of the androgen receptor in prostate myofibroblasts. Mol Cell Endocrinol 2014;384:185199.

8 Zhang Y, Zhang XO, Chen T, Xiang JF, Yin QF, Xing YH, Zhu S, Yang L, Chen LL: Circular intronic long noncoding RNAs. Mol Cell 2013;51:792-806.

-9 Arnberg AC, Van Ommen GJ, Grivell LA, Van Bruggen EF, Borst P: Some yeast mitochondrial RNAs are circular. Cell 1980;19:313-319.

$>10$ Hsu MT, Coca-Prados M: Electron microscopic evidence for the circular form of RNA in the cytoplasm of eukaryotic cells. Nature 1979;280:339-340.

-11 Matsumoto Y, Fishel R, Wickner RB: Circular single-stranded RNA replicon in Saccharomyces cerevisiae. Proc Natl Acad Sci U S A 1990;87:7628-7632.

12 Salzman J, Gawad C, Wang PL, Lacayo N, Brown PO: Circular RNAs are the predominant transcript isoform from hundreds of human genes in diverse cell types. PLoS One 2012;7:e30733.

13 Romero Otero J, Garcia Gomez B, Campos Juanatey F, Touijer KA: Prostate cancer biomarkers: an update. Urol Oncol 2014;32:252-260.

14 Zhang XO, Wang HB, Zhang Y, Lu X, Chen LL, Yang L: Complementary sequence-mediated exon circularization. Cell 2014;159:134-147.

15 Rong D, Tang W, Li Z, Zhou J, Shi J, Wang H, Cao H: Novel insights into circular RNAs in clinical application of carcinomas. Onco Targets Ther 2017;10:2183-2188.

16 Kristensen LS, Hansen TB, Veno MT, Kjems J: Circular RNAs in cancer: opportunities and challenges in the field. Oncogene 2018;37:555-565.

-17 Zhou LH, Yang YC, Zhang RY, Wang P, Pang MH, Liang LQ: CircRNA_0023642 promotes migration and invasion of gastric cancer cells by regulating EMT. Eur Rev Med Pharmacol Sci 2018;22:2297-2303.

-18 Chen L, Zhang S, Wu J, Cui J, Zhong L, Zeng L, Ge S: circRNA_100290 plays a role in oral cancer by functioning as a sponge of the miR-29 family. Oncogene 2017;36:4551-4561.

19 Zhu X, Wang X, Wei S, Chen Y, Chen Y, Fan X, Han S, Wu G: hsa_circ_0013958: a circular RNA and potential novel biomarker for lung adenocarcinoma. Febs j 2017;284:2170-2182.

20 Zhang Y, Liu H, Li W, Yu J, Li J, Shen Z, Ye G, Qi X, Li G: CircRNA_100269 is downregulated in gastric cancer and suppresses tumor cell growth by targeting miR-630. Aging (Albany NY) 2017;9:1585-1594.

21 Lu L, Sun J, Shi P, Kong W, Xu K, He B, Zhang S, Wang J: Identification of circular RNAs as a promising new class of diagnostic biomarkers for human breast cancer. Oncotarget 2017;8:44096-44107.

22 Qu S, Liu Z, Yang X, Zhou J, Yu H, Zhang R, Li H: The emerging functions and roles of circular RNAs in cancer. Cancer Lett 2018;414:301-309.

23 Liao Q Wang J, Pei Z, Xu J, Zhang X: Identification of miRNA-mRNA crosstalk in CD4(+) T cells during HIV-1 infection by integrating transcriptome analyses. J Transl Med 2017;15:41. 


\section{Cellular Physiology Cell Physiol Biochem 2018:50:1903-1915

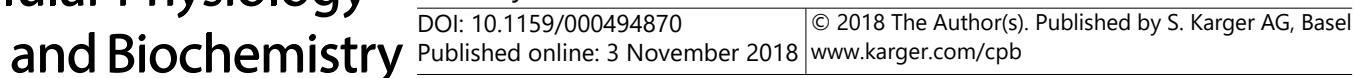 \\ Xia et al.: circRNAs Profiling in Prostate Cancer}

24 Gottipati S, Warncke J, Vollmer R, Humphrey PA: Usual and unusual histologic patterns of high Gleason score 8 to 10 adenocarcinoma of the prostate in needle biopsy tissue. Am J Surg Pathol 2012;36:900-907.

25 Pastuszak AW, Pearlman AM, Godoy G, Miles BJ, Lipshultz LI, Khera M: Testosterone replacement therapy in the setting of prostate cancer treated with radiation. Int J Impot Res 2013;25:24-28.

-26 Jansen FH, van Schaik RH, Kurstjens J, Horninger W, Klocker H, Bektic J, Wildhagen MF, Roobol MJ, Bangma $\mathrm{CH}$, Bartsch G: Prostate-specific antigen (PSA) isoform p2PSA in combination with total PSA and free PSA improves diagnostic accuracy in prostate cancer detection. Eur Urol 2010;57:921-927.

27 Park JY, Yoon G: Overexpression of Aquaporin-1 is a Prognostic Factor for Biochemical Recurrence in Prostate Adenocarcinoma. Pathol Oncol Res 2017;23:189-196.

-28 Petersen LF, Brockton NT, Bakkar A, Liu S, Wen J, Weljie AM, Bismar TA: Elevated physiological levels of folic acid can increase in vitro growth and invasiveness of prostate cancer cells. BJU Int 2012;109:788-795.

29 Hultdin J, Van Guelpen B, Bergh A, Hallmans G, Stattin P: Plasma folate, vitamin B12, and homocysteine and prostate cancer risk: a prospective study. Int J Cancer 2005;113:819-824.

-30 Tisman G, Garcia A: Control of prostate cancer associated with withdrawal of a supplement containing folic acid, L-methyltetrahydrofolate and vitamin B12: a case report. J Med Case Rep 2011;5:413.

31 Vitiello M, Tuccoli A, Poliseno L: Long non-coding RNAs in cancer: implications for personalized therapy. Cell Oncol (Dordr) 2015;38:17-28.

32 Rysman E, Brusselmans K, Scheys K, Timmermans L, Derua R, Munck S, Van Veldhoven PP, Waltregny D, Daniels VW, Machiels J, Vanderhoydonc F, Smans K, Waelkens E, Verhoeven G, Swinnen JV: De novo lipogenesis protects cancer cells from free radicals and chemotherapeutics by promoting membrane lipid saturation. Cancer Res 2010;70:8117-8126.

33 Fritz V, Benfodda Z, Rodier G, Henriquet C, Iborra F, Avances C, Allory Y, de la Taille A, Culine S, Blancou H, Cristol JP, Michel F, Sardet C, Fajas L: Abrogation of de novo lipogenesis by stearoyl-CoA desaturase 1 inhibition interferes with oncogenic signaling and blocks prostate cancer progression in mice. Mol Cancer Ther 2010;9:1740-1754.

-34 Deep G, Schlaepfer IR: Aberrant Lipid Metabolism Promotes Prostate Cancer: Role in Cell Survival under Hypoxia and Extracellular Vesicles Biogenesis. Int J Mol Sci 2016;17. pii: E1061.

-35 Swinnen JV, Van Veldhoven PP, Esquenet M, Heyns W, Verhoeven G: Androgens markedly stimulate the accumulation of neutral lipids in the human prostatic adenocarcinoma cell line LNCaP. Endocrinology 1996;137:4468-4474.

-36 Swinnen JV, Brusselmans K, Verhoeven G: Increased lipogenesis in cancer cells: new players, novel targets. Curr Opin Clin Nutr Metab Care 2006;9:358-365. 